Article

\title{
Tunable Plasmonic Band-Pass Filter with Dual Side-Coupled Circular Ring Resonators
}

\author{
Dongdong Liu ${ }^{1,2}$, Jicheng Wang ${ }^{3, *}$, Feng Zhang ${ }^{4}$, Yuewu Pan ${ }^{2}$, Jian Lu ${ }^{1, *}$ and Xiaowu Ni ${ }^{1}$ \\ 1 School of Science, Nanjing University of Science \& Technology, Nanjing 210094, China; \\ gnod6@sohu.com (D.L.); jsnjnxw@gmail.com (X.N.) \\ 2 School of Mathematics \& Physics Science, Xuzhou University of Technology, Xuzhou 221018, China; \\ yuewupan@163.com \\ 3 School of Science, Jiangsu Provincial Research Center of Light Industrial Optoelectronic Engineering \\ and Technology, Jiangnan University, Wuxi 214122, China \\ 4 Key Laboratory of Semiconductor Materials Science, Institute of Semiconductors, \\ Chinese Academy of Sciences, Beijing 100083, China; fzhang@semi.ac.cn \\ * Correspondence: jcwang@jiangnan.edu.cn (J.W.); lujian@mail.njust.edu.cn (J.L.); \\ Tel.: +86-187-615-23082 (J.W.); +86-139-519-45467 (J.L.)
}

Academic Editor: Vittorio M. N. Passaro

Received: 28 January 2017; Accepted: 7 March 2017; Published: 13 March 2017

\begin{abstract}
A wavelength band-pass filter with asymmetric dual circular ring resonators in a metal-insulator-metal (MIM) structure is proposed and numerically simulated. For the interaction of the local discrete state and the continuous spectrum caused by the side-coupled resonators and the baffle, respectively, the transmission spectrum exhibits a sharp and asymmetric profile. By adjusting the radius and material imbedded in one ring cavity, the off-to-on plasmon-induced absorption (PIA) optical response can be tunable achieved. In addition, the structure can be easily extended to other similar compact structures to realize the filtering task. Our structures have important potential applications for filters and sensors at visible and near-infrared regions.
\end{abstract}

Keywords: integrated optics devices; waveguides; plasmonics; metal-insulator-metal (MIM); sensors

\section{Introduction}

Surface plasmon polaritons (SPPs), a kind of transverse electromagnetic wave tightly confined at the interface between metal and dielectric material, is able to propagate up to a few micrometers [1,2]. Their outstanding ability for overcoming the classical optical diffraction limit has made SPPs attractive as energy and information carriers in highly integrated optical circuits and devices [3,4].

Among various SPP structures, insulator-metal-insulator (IMI) structures and metal-insulator-metal (MIM) structures are two important multilayer plasmonic structures. Due to not only supporting modes with deep sub-wavelength scales and high group velocity over a very wide range of frequencies but also offering very high optical confinement and acceptable propagation length [5], metal-insulator-metal (MIM) structures, such as optical filters [6-9], optical switches [10], demultiplexers [11,12], and sensors [13-16], are widely used. Plasmonic filters based on MIM waveguide structures, such as asymmetric nanodisk filter and sensor [17-19], side-coupled cavity sensor [20], notch resonator filter and sensor [21], and circular ring filter and sensor [22,23], are one of the most important optical devices, have attracted tremendous attention, and have been investigated widely in recent years. All of the above-mentioned devices are promising candidates for highly integrated optical circuits. Electromagnetically induced transparency (EIT), a quantum mechanical phenomenon, reduces light absorption over a narrow spectral region in a coherently driven atomic system [24]. However, hard operation conditions limit its development, and then alternative plasmon-induced transparency (PIT) effects that are 
analogous to the EIT effects, are demonstrated in various MIM waveguide structures [25-27]. Recently, plasmon-induced absorption (PIA), an opposite effect of the PIT effect, have been widely studied and demonstrated in the MIM structures [28-30]. Especially, PIA response is investigated in a MIM-based end-coupled composite-slot-cavity resonator [31]. These MIM structures will be beneficial for optical switching in highly integrated photonic devices.

In this paper, a wavelength filter consisting of dual side-coupled circular ring resonators and two waveguides with a baffle is proposed. Numerical simulation by finite element method (FEM) was conducted to analyze our designs. The results show PIA transmission in spectra. By changing the radius or the refractive index of one ring, we achieve an on-to-off PIA optical response. Moreover, similar structures were designed by moving the location of one ring of the above system. The corresponding transmission spectra and the magnetic intensity distributions $\left|H_{z}\right|^{2}$ at special wavelengths were investigated.

\section{Model and Theoretical Analysis}

Multiple ring resonators are widely used in optical filtering. Here we used two ring resonators to investigate the design consideration about the optical filter. The proposed structure, shown schematically in Figure 1, consists of two ring resonators and two waveguides with a baffle (the blue area). The system is a two-dimension model. The FEM with COMSOL Multiphysics is employed to realize our simulations. The calculated area is divided by Yee's mesh with a size of $2 \mathrm{~nm}$. The FEM with scattering boundary condition is employed to investigate the transmission characteristics of the structure. Two MIM waveguides are marked as I and II. Port 1 and Port 2 are input and output ports, respectively. We assumed the media inside the rings and waveguides to be air (the white area in Figure 1). The widths of the waveguides and the rings, both $w$, are fixed at $50 \mathrm{~nm}$. Since the widths $w$ is much smaller than the wavelength of the incident light, only the fundamental plasmonic mode $\mathrm{TM}_{0}$ could exist in the structure. The $\mathrm{TM}_{0}$-polarized plane wave launched to the left waveguide is used for exciting SPP waves, which is indicated by the arrow in Figure 1. The outer (inner) radii of the two rings are $r_{1}\left(r_{2}\right)$ and $r_{1}{ }^{\prime}\left(r_{2}{ }^{\prime}\right)$, respectively. Meanwhile, we defined $r=\left(r_{1}+r_{2}\right) / 2$ and $r^{\prime}=\left(r_{1}{ }^{\prime}+r_{2}{ }^{\prime}\right) / 2$ as the radii of two ring resonators; $t$ is the coupling distance between the waveguide and the ring; $g$ is the thickness of the baffle. The metal is set as silver, whose frequency-dependent dielectric constant is given by the well-known Drude model [5]:

$$
\varepsilon_{m}(\omega)=\varepsilon_{\infty}-\frac{\omega_{p}^{2}}{\omega(\omega+i \gamma)}
$$

where $\varepsilon_{\infty}=3.7$ is the dielectric constant at the infinite frequency, $\gamma=2.73 \times 10^{13} \mathrm{~Hz}$ is the electron collision frequency, $\omega_{p}=1.38 \times 10^{16} \mathrm{~Hz}$ is the bulk plasma frequency, and $\omega$ stands for the angular frequency of the incident electromagnetic radiation. The propagation constant $\beta$ of SPPs is determined by the following equation [5]:

$$
\tanh \left(\frac{w \sqrt{\beta^{2}-k_{0}^{2} \varepsilon_{i}}}{2}\right)=\frac{-\varepsilon_{i} \sqrt{\beta^{2}-k_{0}^{2} \varepsilon_{m}(\omega)}}{\varepsilon_{m}(\omega) \sqrt{\beta^{2}-k_{0}^{2} \varepsilon_{i}}}
$$

where $\varepsilon_{m}$ and $\varepsilon_{i}$ are the dielectric constants of the silver and air, respectively. $k_{0}$ is the wave vector of light in vacuum. The effective refractive index follows $n_{\text {eff }}=\beta / k_{0}$. The real part of $n_{\text {eff }}$ as a function of $w$ and $\lambda$ is shown in Figure 2. For a fixed wavelength, $\operatorname{Re}\left(n_{\text {eff }}\right)$ gradually grows as wavelength $\lambda$ increases. Theoretically, the resonant wavelength of the ring resonator can be derived from the equation:

$$
\frac{J_{n \prime}^{\prime}\left(k r_{1}\right)}{J_{n^{\prime}}^{\prime}\left(k r_{2}\right)}=\frac{N_{n \prime}^{\prime}\left(k r_{1}\right)}{N_{n \prime}^{\prime}\left(k r_{2}\right)}
$$


where $k=\omega\left(\varepsilon_{0} \varepsilon_{\gamma} \mu_{0}\right)^{1 / 2}$, and $\varepsilon_{0}$ and $\mu_{0}$ are the dielectric constant and permeability in vacuum, respectively. $\varepsilon_{\gamma}=\left(n_{e f f}\right)^{2} / \mu_{0}$ is the frequency-dependent effective relative permittivity. $J_{n \prime}$ is the Bessel function of the first kind with order $n^{\prime}$, and $N_{n}$ is the Bessel function of the second kind with order $n^{\prime} . J_{n^{\prime}}^{\prime}$ and $N_{n \prime}^{\prime}$ are the derivatives of the Bessel functions to the argument $k r$. The resonant wavelength also satisfies the simple relation $\operatorname{Re}\left(n_{\text {eff }}\right) L=m \lambda$, where $m$ is the resonant mode number, a positive integer.

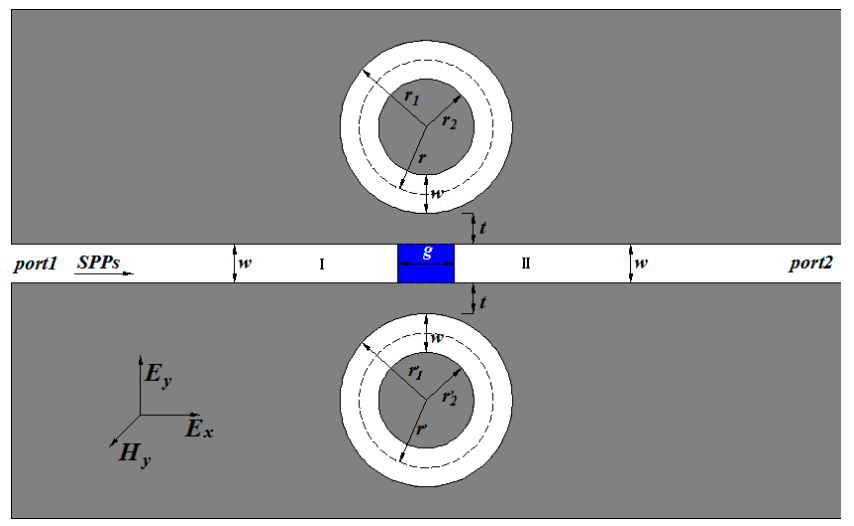

Figure 1. Schematic of the structure composed of two shunt-wound rings resonators with two waveguides and the structure parameters are $w=50 \mathrm{~nm}, g=20 \mathrm{~nm}$, and $t=10 \mathrm{~nm}$.

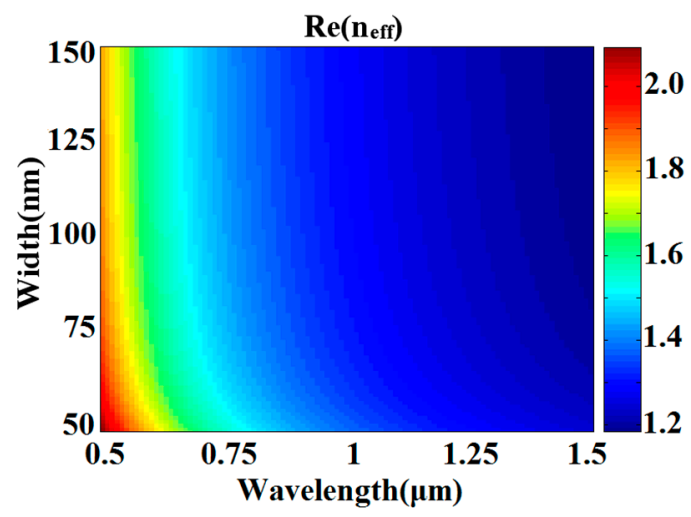

Figure 2. Real part of the effective refractive index $n_{\text {eff }}$ versus the incident wavelength $\lambda$ and the width $w$ in MIM waveguide.

\section{Results and Discussion}

Firstly, we focused on this two shunt-wound rings system, shown in Figure 1. The baffle is assumed to be silver. In this case, the two rings cannot interact with each other. Therefore, one portion of SPPs coupled to the upper ring, the other to the lower ring, both of them will interference with the third portion, which pass through the baffle. At first, the influence of the radius $r^{\prime}$ of the lower ring was discussed. We set $r^{\prime}$ as from $225 \mathrm{~nm}$ to $209 \mathrm{~nm}$ at the step of $4 \mathrm{~nm}$. Other geometric parameters were chosen as follows: $r=225 \mathrm{~nm}, t=10 \mathrm{~nm}$, and $g=20 \mathrm{~nm}$. The simulation results are plotted in Figure 3a. Figure 3a clearly shows that, when $r=r^{\prime}=225 \mathrm{~nm}$, sharp and asymmetrical spectral profiles, which are regarded as Fano resonances [32,33], can be obtained. Here, Fano resonances result from the interference of the broad spectrum and the discrete resonance, which are caused by the baffle and the ring cavities, respectively. As $r^{\prime}$ decreases, it is obviously observed that the transmittance peak of each mode splits into two and the dips at different modes are becoming more apparent. Moreover, the left peaks of each mode exhibit a blue shift (denoted by the blue dotted line) with $r^{\prime}$ decreasing, and the right keep almost unchanged because $r$ is fixed to $225 \mathrm{~nm}$. It goes without saying that the left peaks 
are determined by the lower ring. Figure 4 shows the corresponding field distributions of $\left|H_{Z}\right|^{2}$ when $r=225 \mathrm{~nm}$ and $r^{\prime}=213 \mathrm{~nm}$. It is observed that the energy of the resonant peaks is confined in only one cavity, the energy of the dip in two rings. These behaviors of the two resonances accord well with the analysis.

(a)

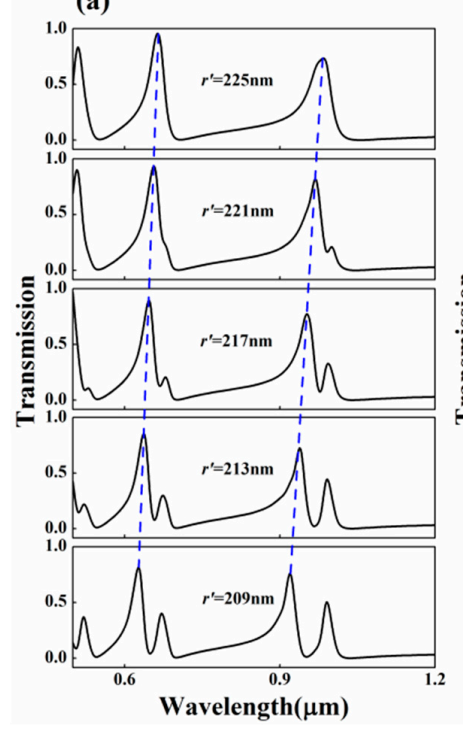

(b)

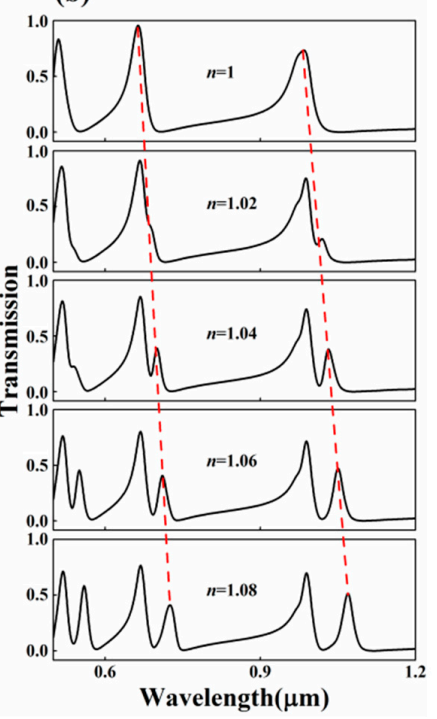

Figure 3. Transmission spectra of two shunt-wound rings system (a) for different radius $r^{\prime}$ of the lower ring; (b) for different refractive index $n$ of the lower ring.

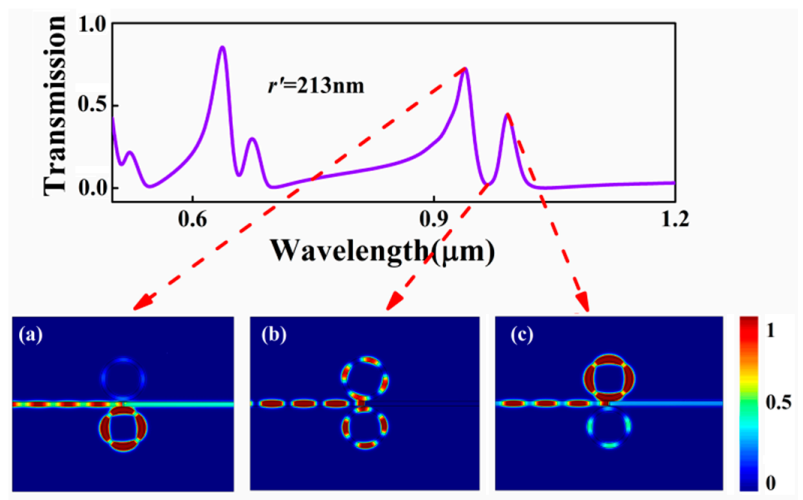

Figure 4. Transmission spectrum of the MIM waveguide with two shunt-wound ring resonators when $r^{\prime}=213 \mathrm{~nm}$; the contour profiles of magnetic intensity distributions $\left|H_{\mathrm{Z}}\right|^{2}$ of the device at (a) $\lambda=940 \mathrm{~nm}$; (b) $\lambda=967 \mathrm{~nm}$; (c) $\lambda=991 \mathrm{~nm}$.

In a similar way, we provided another way to control the transmission windows by changing the refractive index $n$ of the lower ring. The refractive index $n$ of the lower ring was set from 1 to 1.08 with a step of 0.02 , and other parameters were kept the same as the above. We plotted the simulated results in Figure $3 \mathrm{~b}$. As $n$ grows, the dips are becoming more obvious and the right peaks of each mode show a little red-shift (denoted by the read dotted line) in the spectra. Similarly, the shifting effect is more apparent and the width of dips is wider at low resonant modes. From the aforementioned analysis, two methods were found to achieve on-to-off PIA response in a two shunt-wound rings filter system.

In addition, the influences of baffle dimension and position on the transmission spectrum were investigated. The results showed that, as $g$ grows, the transmission peaks gradually lower; when the baffle shift to the right or the left is less than $150 \mathrm{~nm}$, the transmission peaks do not show a shift in the $\mathrm{x}$-axis; when the shifting distance is more than $150 \mathrm{~nm}$, little energy is transmitted. 
Successively, in addition to the above case, the structure proposed in Figure 1 can be also extended to another situation, the MIM bus waveguide side-coupled two series-wound rings, shown in Figure 5. The separation between two rings is set as $10 \mathrm{~nm}$ and the radii of the two rings are all set as $225 \mathrm{~nm}$; other parameters are kept the same as above. Due to the side-coupled arrangements, the bottom ring cavity couples strongly to the bus waveguide, the so-called bright mode; the upper ring cavity cannot directly couple to the bus waveguide, known as the dark mode. On account of the greatly enhanced near-field interferences between two rings, the dark mode is excited through tunneling coupling with the bright mode. As a result, PIA transmission appears at the pass band of the structure without the upper ring cavity, with two new resonant peaks generated as shown in Figure 5. The corresponding magnetic intensity distributions $\left|H_{\mathrm{z}}\right|^{2}$ at the two peaks and the one dip for the second-order mode are displayed in Figure 6a-c. Obviously, unlike shunt-wound rings system, for transmission peaks, the bottom ring is directly excited by the incident SPPs, and the upper ring is also enhanced thanks to the coupling with the bottom ring resonator. The in-phase coupling resonance occurs at the two rings with the wavelength of $\lambda=961 \mathrm{~nm}$, shown in the inset of Figure $6 \mathrm{a}$, whereas the out-phase forms at the wavelength of $\lambda=1027 \mathrm{~nm}$ shown in the inset of Figure 6c. The incident SPP power can be coupled into the right waveguide by the bottom ring, leading to the newly appeared transmission peaks. For the dip, however, the dark resonator is efficiently activated, whereas the bright resonator is extraordinarily suppressed due to the strong destructive interference. Almost all SPP power is absorbed in the upper ring cavity, and little SPP power is coupled into the right MIM waveguide output shown in Figure 6b, which is direct evidence of the remarkable absorption window in the intrinsic peak point. Hence, the introduced upper ring cavity can store all SPP power and achieve PIA response.

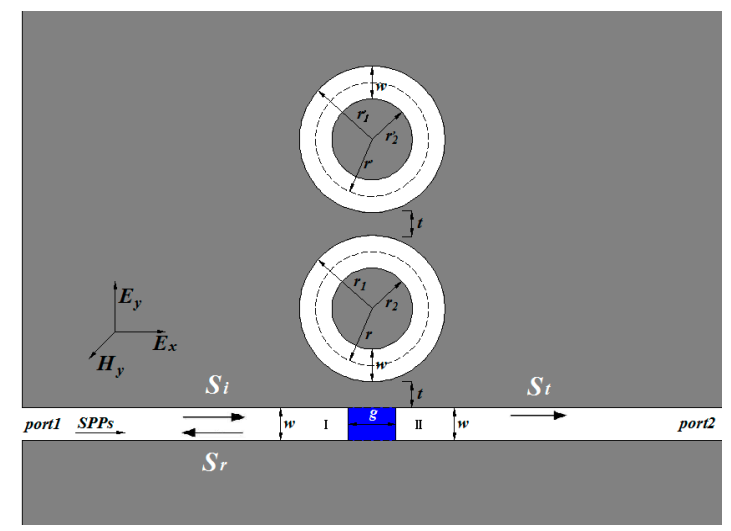

Figure 5. Schematic of the structure composed of two series-wound ring resonators with two waveguides.

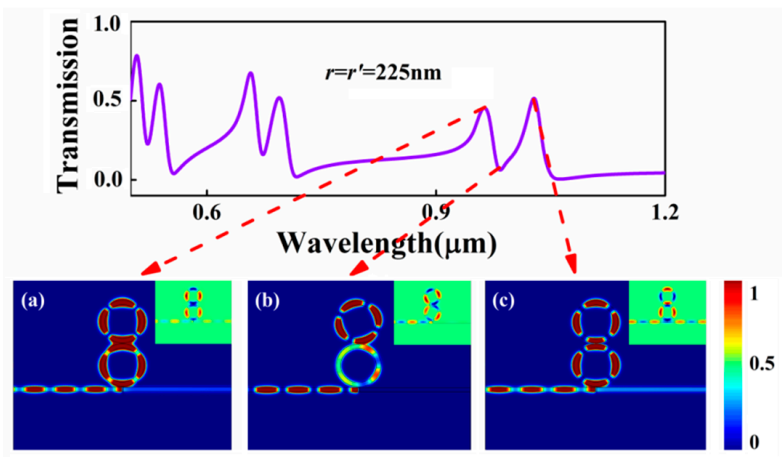

Figure 6. Transmission spectrum of the MIM waveguide with two series-wound ring resonators when $r=r^{\prime}=225 \mathrm{~nm}$; the contour profiles of magnetic intensity distributions $\left|H_{\mathrm{Z}}\right|^{2}$ of the device at (a) $\lambda=961 \mathrm{~nm}$; (b) $\lambda=986 \mathrm{~nm}$; (c) $\lambda=1027 \mathrm{~nm}$. The insets denote the corresponding $H_{\mathrm{z}}$ distribution of SPPs in the PIT systems. 
On the other hand, the transmission properties can be analyzed by the temporal coupled mode theory (CMT) [34,35]. As shown in Figure 5, the amplitudes of the incident, reflected, and total-transmitted SPP waves are denoted by $S_{i}, S_{r}$, and $S_{t}$, respectively, and are normalized to the power in the modes. $Q_{r}$ is cavity quality factors related to intrinsic loss in the dual rings; $Q_{w}$ is the quality factor related to the coupling loss between waveguides and the bottom ring; $Q_{c}$ is the quality factor related to the coupling loss between two rings. The time evolution normalized amplitude $a_{1}$ of the bottom ring and $a_{2}$ of the upper ring can be described from the CMT:

$$
\begin{gathered}
\frac{d a_{1}}{d t}=\left(j \omega_{0}-\frac{\omega_{0}}{2 Q_{r}}-\frac{\omega_{0}}{2 Q_{w}}\right) a_{1}+\sqrt{\frac{\omega_{0}}{Q_{w}}} S_{i}-j \frac{\omega_{0}}{2 Q_{c}} a_{2} \\
\frac{d a_{2}}{d t}=\left(j \omega_{0}-\frac{\omega_{0}}{2 Q_{r}}\right) a_{2}-j \frac{\omega_{0}}{2 Q_{c}} a_{1}
\end{gathered}
$$

where $\omega_{0}$ is the same resonant frequency of the dual rings. According to energy conservation, the amplitude of the input and the output lights in the waveguide should satisfy the following relationships:

$$
\begin{gathered}
S_{r}=-S_{i}+j \sqrt{\frac{\omega_{0}}{Q_{w}}} a_{1} \\
S_{t}=j \sqrt{\frac{\omega_{0}}{Q_{w}}} a_{1} .
\end{gathered}
$$

Therefore, the transmission coefficient $T$ can be obtained as follows:

$$
T=\left|\frac{S_{t}}{S_{i}}\right|^{2}=\left|\frac{2}{Q_{w}} \frac{j 2\left(\frac{\omega-\omega_{0}}{\omega_{0}}\right)+\frac{1}{Q_{r}}}{\left(j 2\left(\frac{\omega-\omega_{0}}{\omega_{0}}\right)+\frac{1}{Q_{r}}+\frac{1}{2 Q_{w}}\right)^{2}+\left(\frac{1}{Q_{c}}\right)^{2}-\left(\frac{1}{2 Q_{w}}\right)^{2}}\right|^{2} .
$$

Finally, the two concentric rings system was investigated. As shown in Figure 7, the radius $r^{\prime}$, width $w^{\prime}$, and refractive index $n^{\prime}$ of the inner ring are set at $158 \mathrm{~nm}, 20 \mathrm{~nm}$, and 1.02, respectively; other parameters are kept the same as the above. The transmission spectrum was calculated and is shown in Figure 7. According to the above theoretical analysis results, for coupling directly to the bus waveguide, the outer ring is called the bright mode, and on the contrary, the inner ring is called the dark mode. Similarly, on account of the greatly enhanced near-field interferences between two rings, it is obvious that two resonant peaks appear in each mode in the transmission spectrum. In order to find out the underlying physics of the resonant peaks in the transmission spectrum, the magnetic intensity distributions $\left|H_{z}\right|^{2}$ at the two peaks and one dip for the second-order mode are displayed in Figure 7a-c. The same as series-wound rings system, for transmission peaks, the outer ring is directly excited by the incident SPPs and the inner ring couples with the outer ring. The in-phase and out-phase coupling resonance occurs between the two rings at $\lambda=898 \mathrm{~nm}$ and $\lambda=1035 \mathrm{~nm}$, respectively, shown in the insets of Figure 7a,c. However, for the dip, due to the strong destructive interference, the bright resonator is suppressed and the dark resonator is efficiently activated. Almost all SPP energy is absorbed in the inner ring cavity, and little SPP energy is coupled into the right MIM waveguide output shown in Figure $7 \mathrm{~b}$. These results provide a theoretical basis for designing highly integrated optical devices. 


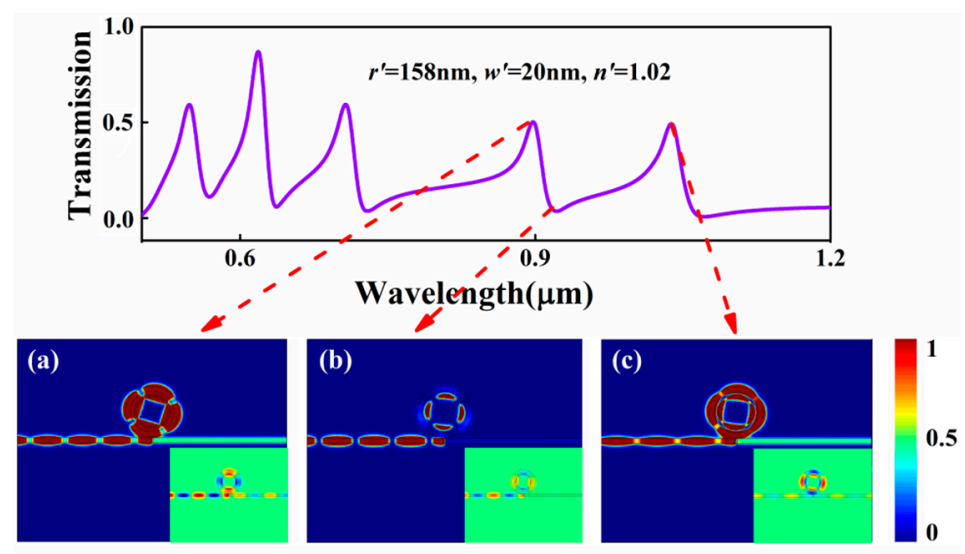

Figure 7. Transmission spectrum of the MIM waveguide with two concentric rings resonators when $r^{\prime}=158 \mathrm{~nm}, w^{\prime}=20 \mathrm{~nm}$, and $n^{\prime}=1.02$; the contour profiles of magnetic intensity distributions $\left|H_{z}\right|^{2}$ of the device at (a) $\lambda=898 \mathrm{~nm}$; (b) $\lambda=921 \mathrm{~nm}$; (c) $\lambda=1035 \mathrm{~nm}$. The insets denote the corresponding $H_{\mathrm{z}}$ distribution of SPPs in the concentric rings system.

\section{Conclusions}

In summary, we studied the transmission characteristics of a wavelength band-pass filter consisting of dual side-coupled circular ring resonators and two waveguides with a baffle. For the two shunt-wound rings system, we provided two approaches to control the transmission spectra. An on-to-off PIA optical response could be achieved by adjusting the radius or the refractive index of the lower ring. We found the shifting effect is more apparent and the width of the absorption dips is wider at low resonant modes. For the series-wound rings system and the concentric rings system, the corresponding transmission spectra and the magnetic intensity distributions $\left|H_{\mathrm{Z}}\right|^{2}$ at special wavelengths were investigated. Compared with the shunt-wound rings system, it is difficult to achieve the on-to-off PIA optical response by adjusting the structural parameters of these two systems although there are PIA windows in the transmission spectra. The analyses make these kinds of experiments easier and pave the way for actively tunable sensoring applications.

Acknowledgments: This work is supported by the National Basic Research Program of China (Grant No. 2015CB759600), the National Natural Science Foundation of China (Grant Nos. 11504139, 61474113 and 61574140), the Natural Science Foundation of Jiangsu Province (Grant No. BK20140167), the Natural Science Foundation of the Jiangsu Higher Education Institutions of China (Grant No. 16KJB140016), the Key Laboratory Open Fund of Institute of Semiconductors of CAS (Grant No. KLSMS-1604), and the Nature Science Foundation of Xuzhou University of Technology of China (Grand No. XKY2014206).

Author Contributions: D.L. and J.W. conceived and designed the simulation; D.L. and Y.P. performed the simulation and analyzed the data; D.L., J.W. and F.Z. wrote the manuscript under the supervision of J.L., D.L., J.W., F.Z. and J.L. supervised the project. All authors contributed to the scientific discussion of the results and reviewed the manuscript.

Conflicts of Interest: The authors declare no conflict of interest.

\section{References}

1. Barnes, W.L.; Dereux, A.; Ebbesen, T.W. Surface plasmon subwavelength optics. Nature 2003, 424, 824-830. [CrossRef] [PubMed]

2. Genet, C.; Ebbesen, T.W. Light in tiny holes. Nature 2007, 445, 39-46. [CrossRef] [PubMed]

3. Neutens, P.; Dorpe, P.V.; Vlaminck, I.D.; Lagae, L.; Borghs, G. Electrical detection of confined gap plasmons in metal-insulator-metal waveguides. Nat. Photonics 2009, 3, 283-286. [CrossRef]

4. Gramotnev, D.K.; Bozhevolnyi, S.I. Plasmonics beyond the diffraction limit. Nat. Photonics 2010, 4, 83-91. [CrossRef]

5. Liu, D.D.; Sun, Y.; Fan, Q.B.; Mei, M.F.; Wang, J.C.; Pan, Y.W.; Lu, J. Tunable plasmonically induced transparency with asymmetric multi-rectangle resonators. Plasmonics 2016, 11, 1621-1628. [CrossRef] 
6. Lin, X.S.; Huang, X.G. Tooth-shaped plasmonic waveguide filters with nanometeric sizes. Opt. Lett. 2008, 33, 2874-2876. [CrossRef] [PubMed]

7. Lee, T.; Lee, D.; Kwon, S. Dual-function metal-insulator-metal plasmonic optical filter. IEEE Photonics J. 2015, 7, 4800108. [CrossRef]

8. Zand, I.; Bahramipanah, M.; Abrishamian, M.S.; Liu, J.M. Metal-insulator-metal nanoscale loop-stub structures. IEEE Photonics J. 2012, 4, 2136-2142. [CrossRef]

9. Lu, H.; Liu, X.M.; Mao, D.; Wang, L.R.; Gong, Y.K. Tunable band-pass plasmonic waveguide filters with nanodisk resonators. Opt. Express 2010, 18, 17922-17927. [CrossRef] [PubMed]

10. Tao, J.; Wang, Q.J.; Huang, X.G. All-optical plasmonic switches based on coupled nano-disk cavity structures containing nonlinear material. Plasmonics 2011, 6, 753-759. [CrossRef]

11. Liu, H.Q.; Gao, Y.X.; Zhu, B.F.; Ren, G.B.; Jian, S.S. A T-shaped high resolution plasmonic demultiplexer based on perturbations of two nanoresonators. Opt. Commun. 2015, 334, 164-169. [CrossRef]

12. Wang, G.X.; Lu, H.; Liu, X.M.; Mao, D.; Duan, L.N. Tunable multi-channel wavelength demultiplexer based on MIM plasmonic nanodisk resonators at telecommunication regime. Opt. Express 2011, 19, 3513-3518. [CrossRef] [PubMed]

13. Wu, T.S.; Liu, Y.M.; Yu, Z.Y.; Peng, Y.W.; Shu, C.G.; Ye, H. The sensing characteristics of plasmonic waveguide with a ring resonator. Opt. Express 2014, 22, 7669-7677. [CrossRef] [PubMed]

14. Lin, Q.; Zhai, X.; Wang, L.L.; Luo, X.; Liu, G.D.; Liu, J.P.; Xia, S.X. A novel design of plasmon-induced absorption sensor. Appl. Phys. Express 2016, 9, 062002. [CrossRef]

15. Qu, S.N.; Song, C.; Xia, X.S.; Liang, X.Y.; Tang, B.J.; Hu, Z.D.; Wang, J.C. Detuned plasmonic Bragg grating sensor based on defect metal-insulator-metal waveguide. Sensors 2016, 16, 784. [CrossRef] [PubMed]

16. Qian, Q.L.; Liang, Y.J.; Liang, Y.; Shao, H.Y.; Zhang, M.L.; Xiao, T.; Wang, J.C. Tunable multiple-step plasmonic Bragg reflectors with graphene-based modulated grating. Sensors 2016, 16, 2039. [CrossRef] [PubMed]

17. Zhan, G.Z.; Liang, R.S.; Liang, H.T.; Luo, J.; Zhao, R.T. Asymmetric band-pass plasmonic nanodisk filter with mode inhibition and spectrally splitting capabilities. Opt. Express 2014, 22, 9912-9919. [CrossRef] [PubMed]

18. Lu, H.; Liu, X.M.; Mao, D. Plasmonic analog of electromagnetically induced transparency in multi-nanoresonator-coupled waveguide systems. Phys. Rev. A 2012, 85, 053803. [CrossRef]

19. Wei, W.; Zhang, X.; Ren, X.M. Plasmonic circular resonators for refractive index sensors and filters. Nona. Res. Lett. 2015, 10, 211. [CrossRef] [PubMed]

20. Xie, Y.Y.; Huang, Y.X.; Zhao, W.L.; Xu, W.H.; He, C. A novel plasmonic sensor based on metal-insulator-metal waveguide with side-coupled hexagonal cavity. IEEE Photonics J. 2015, 7, 4800612. [CrossRef]

21. Bahramipanah, M.; Abrishamian, M.S.; Mirtaheri, S.A.; Liu, J.M. Ultracompact plasmonic loop-stub notch filter and sensor. Sens. Actuators B 2014, 194, 311-318. [CrossRef]

22. Zou, S.W.; Wang, F.Q.; Liang, R.S.; Xiao, L.P.; Hu, M. A nanoscale refractive index sensor based on asymmetric plasmonic waveguide with a ring resonator: A review. IEEE Sens. J. 2015, 15, 646-650. [CrossRef]

23. Zhang, H.Y.; Shen, D.L.; Zhang, Y.P. Circular split-ring core resonators used in nanoscale metal-insulator-metal band-stop filters. Laser Phys. Lett. 2014, 11, 115902. [CrossRef]

24. Zhang, S.; Genov, D.A.; Wang, Y.; Liu, M.; Zhang, X. Plasmon-induced transparency in metamaterials. Phys. Rev. Lett. 2008, 101, 047401. [CrossRef] [PubMed]

25. Huang, B.; Meng, H.Y.; Wang, Q.H.; Wang, H.H.; Zhang, X.; Yu, W.; Tan, C.H.; Huang, X.G.; Wang, F.Q. Plasmonic-induced transparency and slow-light effect based on stub waveguide with nanodisk resonator. Plasmonics 2016, 11, 543-550. [CrossRef]

26. He, Z.; Li, H.; Zhan, S.; Cao, G.; Li, B. Combined theoretical analysis for plasmon-induced transparency in waveguide systems. Opt. Lett. 2014, 39, 5543-5546. [CrossRef] [PubMed]

27. Liu, D.D.; Fan, Q.B.; Mei, M.F.; Wang, J.C.; Pan, Y.W.; Teng, D.X.; Lu, J. Tunable multiple plasmon-induced transparency with side-coupled rectangle cavities. Chin. Opt. Lett. 2016, 14, 052302.

28. Wen, K.H.; Hu, Y.H.; Chen, L.; Zhou, J.Y.; He, M.; Lei, L.; Meng, Z.M. Plasmonic-Induced Absorption and Transparency Based on a Compact Ring-Groove Joint MIM Waveguide Structure. IEEE Photonics J. 2016, 8, 4802308. [CrossRef]

29. Li, H.J.; Wang, L.L.; Zhai, X. Plasmonically induced absorption and transparency based on MIM waveguides with concentric nanorings. IEEE Photonics Technol. Lett. 2016, 28, 1454-1457. [CrossRef]

30. Liu, D.D.; Pan, Y.W.; Sun, Y.; Xia, X.S.; Wang, J.C.; Lu, J. Tunable multimode electromagnetically induced absorption transmission in metal-insulator-metal resonators. AIP Adv. 2016, 6, 025219. [CrossRef] 
31. Wen, K.H.; Hu, Y.H.; Zhou, J.Y.; Lei, L.; Li, J.F.; Wu, Y.J. Plasmonic-induced absorption in an end coupled metal-insulator-metal resonator structure. Opt. Mater. Express 2017, 7, 433-443. [CrossRef]

32. Wen, K.; Hu, Y.; Chen, L.; Zhou, J.; Lei, L.; Guo, Z. Fano resonance with ultra-high figure of merits based on plasmonic metal-insulator-metal waveguide. Plasmonics 2015, 10, 27-32. [CrossRef]

33. Wen, K.; Hu, Y.; Chen, L.; Zhou, J.; Lei, L.; Meng, Z. Single/dual Fano resonance based on plasmonic metal-dielectric-metal waveguide. Plasmonics 2016, 11, 315-321. [CrossRef]

34. Haus, H.; Huang, W.P. Couple-mode theory. Proc. IEEE 1991, 79, 1505-1518. [CrossRef]

35. Li, Q.; Wang, T.; Su, Y.; Yan, M.; Qiu, M. Coupled mode theory analysis of mode-splitting in coupled cavity system. Opt. Express 2010, 18, 8367-8382. [CrossRef] [PubMed]

(C) 2017 by the authors. Licensee MDPI, Basel, Switzerland. This article is an open access article distributed under the terms and conditions of the Creative Commons Attribution (CC BY) license (http://creativecommons.org/licenses/by/4.0/). 